\title{
Estrogens, Aging, and Working Memory
}

\author{
Elizabeth Hampson ${ }^{1}$
}

Published online: 11 October 2018

(C) The Author(s) 2018

\begin{abstract}
Purpose of Review Working memory (WM) is a key process that is integral to many complex cognitive tasks, and it declines significantly with advancing age. This review will survey recent evidence supporting the idea that the functioning of the WM system in women is modulated by circulating estrogens.

Recent Findings In postmenopausal women, increased estrogen concentrations may be associated with improved WM function, which is evident on WM tasks that have a high cognitive load or significant manipulation demands. Experimental studies in rhesus monkeys and human neuroimaging studies support a prefrontal locus for these effects. Defining the basic neurochemical or cellular mechanisms that underlie the ability of estrogens to regulate WM is a topic of current research in both human and animal investigations.

Summary An emerging body of work suggests that frontal executive elements of the WM system are influenced by the circulating estrogen concentrations currently available to the CNS and that the effects are region-specific within the frontal cortex. These findings have implications for women's brain health and cognitive aging.
\end{abstract}

Keywords Working memory $\cdot$ Frontal cortex $\cdot$ Estrogen $\cdot$ Estradiol $\cdot$ Short-term memory $\cdot$ Menopause

\section{Introduction}

It is well-established that working memory declines during normal and pathological aging $[1,2]$. The term "working memory" (WM) refers to a short-term storage and control system that underlies the ability to temporarily hold information "online" or manipulate it within memory over time frames of up to a few minutes $\left[2,3^{\bullet}\right]$. It is measured by the delayed response task in nonhuman primates [3•] or by analogous tasks in humans such as the $N$-back task $[4,5]$. Unlike episodic memory, a form of long-term memory that recruits the hippocampus and surrounding temporal cortex, WM is supported by a core network of frontoparietal sites that can be observed by using functional neuroimaging techniques $[1,6]$ (e.g., functional magnetic resonance imaging or fMRI). Damage to particular sites within this network or developmental anomalies that

This article is part of the Topical Collection on Sex and Gender Issues in Behavioral Health

Elizabeth Hampson

ehampson@uwo.ca

1 Department of Psychology, Social Sciences Center, and Department of Psychiatry, University of Western Ontario, London, ON N6A 5C2, Canada interfere with their function causes WM deficits that can be detected by clinical tests [7]. The dorsolateral prefrontal cortex (DLPFC) is a region of particular importance for the performance of WM tasks that require manipulating information currently held in short-term store [7] (e.g., mental arithmetic), as opposed to its simple retention. Although WM is also studied in rodents, the tasks used to study rats or mice often prominently recruit the hippocampal region, not frontal sites, and accordingly, this review will focus on the modulatory role of estrogens in the WM system in humans and nonhuman primates (NHP).

An age-related decline in WM function has long been recognized in both men and women, and is considered a component of normal aging [2]. Indeed, the types of memory most affected by aging are WM and episodic memory $[1,2,4]$, and age-associated atrophy is the most prominent at prefrontal sites [1]. Memory complaints are also commonly reported in association with menopause in women [8,9]. Only recently, however, have researchers begun to conceptualize menopause-related memory concerns as a WM phenomenon. Our laboratory was the first to show an association between the use of estrogens in postmenopausal women and WM performance [10], followed soon after by other labs [11; for review, see $[9,12]$. The initial studies used cross-sectional designs, but the hypothesis that circulating estrogen levels modulate the WM system has now been supported by several other 
paradigms (see below). Early work in primates, carried out in parallel with our own original study, pointed to the same conclusion [13]. Since then, and increasingly during the last 1015 years, human studies together with work in NHP have yielded convergent evidence that estrogens play a permissive role in the functioning of the WM system in the adult female brain. This influential concept has a number of implications for cognitive aging.

Before discussing recent developments, it is important to clarify that hormonal regulation of a specific function does not map in any simple way onto the possibility that a sex difference might exist in the same function, at least in younger adults. Sex differences in WM have had limited direct study. A female advantage has been reported on certain WM tasks in young adults including the spatial working memory (SPWM) task of Duff and Hampson [14] (a task that requires the active holding and recurrent updating of spatial positions within WM) (Fig. 1), digit randomization, the double-span task, as well as, in NHP, the classic delayed response task [12, 14-18], but a female advantage has been observed only when executive demands on WM are high and is not seen on simple spancapacity tasks (e.g., forward digit span) that lack a requirement for sustained mental manipulation of information and emphasize instead the passive storage elements of the WM system that are associated with perisylvian cortex [19]. Conversely, the $N$-back task is a WM task well suited to functional imaging studies, but it shows no, or even a reversed sex difference [20•, $21 \cdot]$, in spite of being subject to estrogen-related modulation at the neural level (see below). Reasons for the inconsistency in the sex difference across different WM tasks are not yet known but might be process-related - the $N$-back task emphasizes the monitoring functions of WM more than manipulation, and its correlations with scores on many other wellestablished WM tests that tap into the executive processes of WM are low [22] (see [12] for discussion). Currently, data suggest that only the executive components of WM, which are regulated by the prefrontal cortex (PFC), are influenced by estradiol levels in women (see below). If true, then sex differences in WM may show age- or hormone-dependent variations in magnitude, a possibility that has yet to be explored through controlled studies (but see [23]).

\section{Working Memory and the Aging Brain}

Substantial evidence from behavioral studies, functional imaging, and cellular/molecular work supports the hypothesis that $17 \beta$-estradiol (the dominant form of estrogen in women of reproductive age) plays a modulatory role in the executive control processes of the frontal WM system. WM changes associated with reduced estradiol production by the ovaries may form at least part of the basis for the decreased memory function subjectively reported by many women as they enter into menopause. In support of this, reduced WM accuracy on the delayed response task is found in female rhesus monkeys following ovariectomy or after the natural menopause and may be restored substantially by exogenous estradiol administration, e.g., [13, 24-26•]. Early work showed that reduced performance on the delayed response task was related to menopause not chronological age [13]. Likewise, a small body of evidence suggests that WM is reduced in postmenopausal women but can be alleviated by estrogen replacement therapies or during a short-term randomized treatment of exogenous $17 \beta$-estradiol [10, 27].

In our own work, we found in a sample of nearly 100 healthy postmenopausal women devoid of other chronic health conditions that those receiving estrogen replacement at menopause performed significantly better than healthy untreated women on three tests of WM, including the SPWM (Fig. 1), irrespective of whether a progestin was also being used [10]. (Although it was a newly developed test when our study was run [14], the SPWM is based on a classic WM task developed by Passingham [28], and accuracy on the early trials of the SPWM correlates moderately to highly with other WM tasks that have a high executive load [e.g., $r=0.52$ (randomized digit ordering), $r=0.58$ (self-ordered pointing task); 12 , 14, 29], demonstrating convergent validity.). Furthermore, the effects in our study were selective - no differences were found on control tests measuring several other cognitive functions, including episodic memory tests that recruit the hippocampal region. Convergent support for our findings first came from an independent study by Keenan et al. [11] in a much smaller sample, who found superior performance on an auditory $N$-back task in postmenopausal women taking estradiol or other estrogens (Premarin) compared with a group of control women not on treatment. Both of these early reports were observational studies. In later work, Krug et al. [27] used a double-blind crossover design to confirm an effect of $17 \beta$-estradiol on a digit randomization test of WM (also used in our own study in 2000) and showed that estradiol's effects extended to a task emphasizing memory for temporal order within episodic memory. Both tasks improved under estradiol treatment compared with placebo. The frontal cortex has been implicated in the ability to correctly remember the temporal ordering of recent events [30]; therefore, the temporal findings, combined with the observation of improved WM under estradiol versus placebo in the context of a controlled trial, solidified the idea of a frontal lobe locus for estradiol's cognitive effects. Notably, Krug et al. found that improvement in WM function was evident after just a 3-day trial of transdermal estradiol, suggesting estradiol's actions on WM have a short onset latency and are not likely attributable to long-term modifications within the CNS. 
Fig. 1 A schematic representation of the spatial working memory (SPWM) task of Duff and Hampson [14]. Participants open two doors at a time on a homogenous appearing background, discovering the colors hidden beneath. Going back to a previously visited pair of locations is considered a working memory error (WME). Top: a matching pair of colors. Second from top: a non-matching pair. Third from top: another non-matching pair. Bottom: an example of a WME. The goal is to find all the matching pairs in as few moves as possible, by opening only two doors at a time. WMEs can be committed on the SPWM in several different ways; full scoring instructions are available upon request from the author. (Reprinted from: Current Opinion in Behavioral Sciences, Vol 23, Hampson, E., Regulation of cognitive function by androgens and estrogens, pp. 49 57,2018 , with permission from Elsevier)
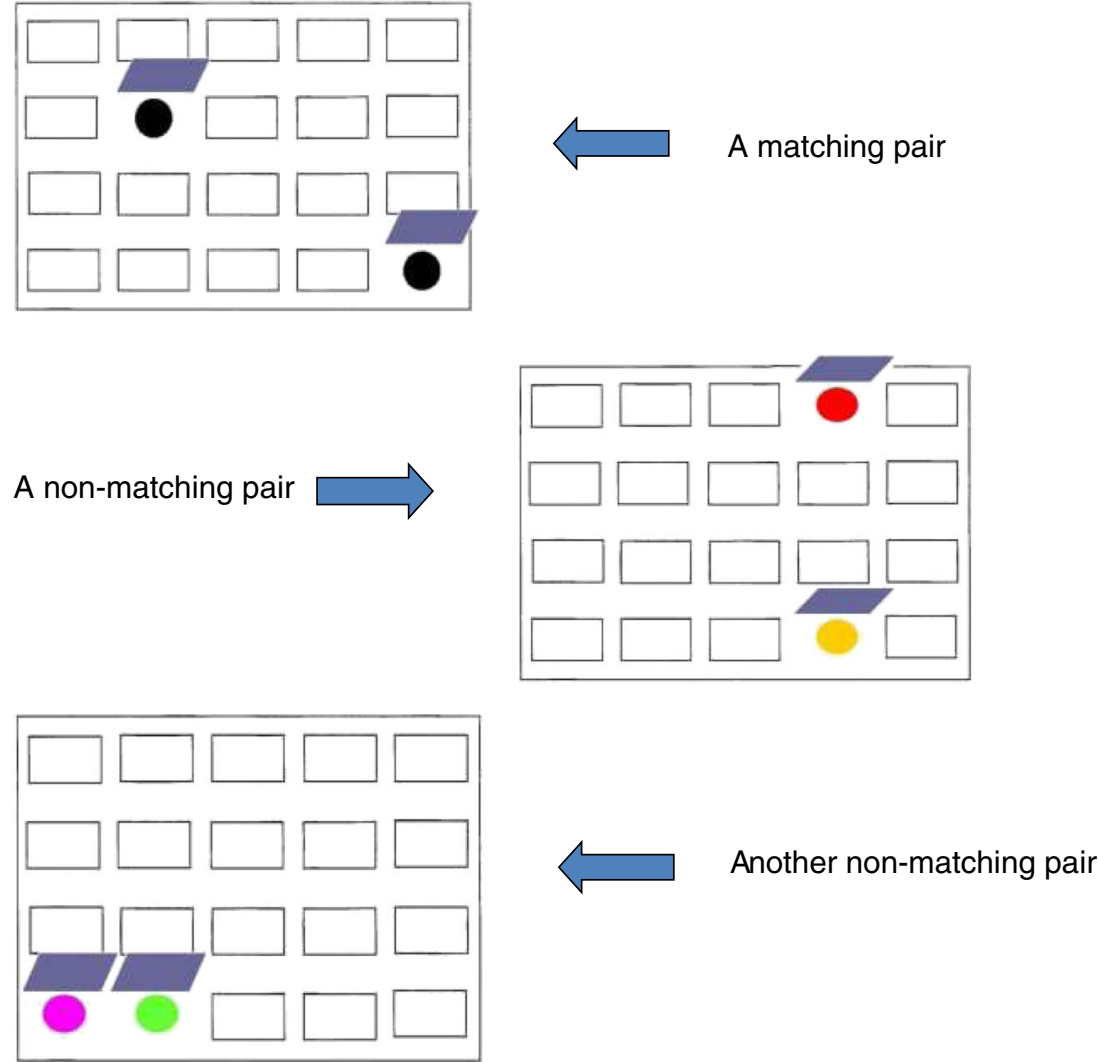

Now, a WME

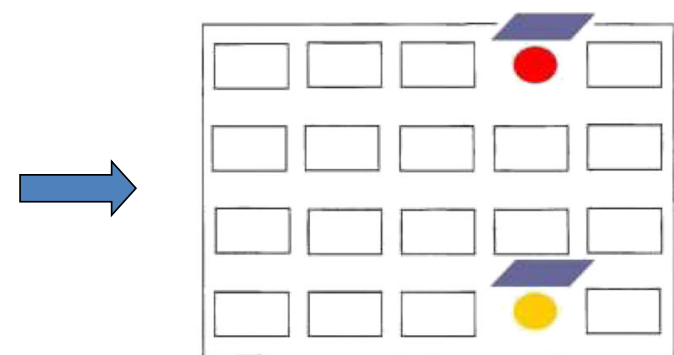

Effects of estrogens on WM are not confined to conditions where the CNS is estrogen-deprived. Most past work on postmenopausal women (or animal models of aging and menopause, e.g., [25]) has indeed focused on conditions characterized by extremely low estrogen availability and on estrogens given exogenously. However, effects on WM have also been reported in younger women. Reduction in endogenous estradiol activity by drugs that inhibit estrogen synthesis (or by tamoxifen, a drug that acts at the estrogen receptor) is reported to diminish $N$-back performance [31, 32], and the consumption of high-dose soy isoflavones containing phytoestrogens led to improved WM during a low-estrogen phase of the menstrual cycle [33]. Conversely, the high concentrations of $17 \beta$ estradiol that characterize the preovulatory or luteal phases of the natural ovarian cycle are associated with modestly improved WM performance on the SPWM [23], compared with the menstrual phase where estradiol production is at its lowest ebb. In a recent study, individual differences in WM accuracy showed a positive correlation with bioavailable estradiol concentrations in women's saliva collected at the time of the cognitive assessment [23]. In contrast, scores on Corsi Blocks were unaffected by estradiol variations over the menstrual cycle in another recent report [34; see also 33].

Whereas cognitive studies speak to the efficient recruitment of the WM system under everyday conditions, functional imaging has been a valuable tool to visualize and confirm a prefrontal locus for the estrogen effects. As expected, sites in the frontal cortex classically associated with working memory processes have been implicated. Functional MRI studies using postmenopausal women as participants have shown altered blood oxygen level-dependent (BOLD) activation in frontal cortex during high load conditions of the $N$-back [35-37] or during other WM tasks [38] when women receive $17 \beta$ estradiol (or ethinyl estradiol) compared with placebo. Smith 
et al. [38], for example, in a placebo-controlled crossover trial of ethinyl estradiol versus placebo found increased BOLD activation under estradiol in prefrontal regions (BA 44 and 45) during a WM task emphasizing active maintenance. A more recent study showed that postmenopausal women not using estrogens resembled men, more than premenopausal women, in task-related connectivity of the DLPFC during an $N$-back task, and the magnitude of BOLD activation in the DLPFC significantly predicted accuracy scores on the WM task [39•]. Since the first demonstrations confirming that circulating levels of estradiol modulate the level of neural activation in the PFC elicited during WM processing, work has turned recently to the complex question of identifying precisely which neurotransmitter mechanisms mediate the WM effects observed (see below).

These human studies have been supplemented by independent research done in NHP, offering convergent support for the human WM findings and also insights into potential molecular mechanisms. It is well known that estradiol can influence neuronal activity in the CNS by binding with high affinity to two forms of the estrogen receptor $(\mathrm{ER} \alpha, \mathrm{ER} \beta)$ that act as transcriptional modulators and at least one membraneassociated receptor (GPER1) capable of mediating rapid non-genomic effects of the steroid [40, 41•]. By acting at its receptors, estradiol drives a wide spectrum of regulatory effects on the neurochemistry and microarchitecture of the adult CNS. Female monkeys display reduced accuracy on delayed response following menopause [13] or after ovariectomy [24] and consistent with estradiol as a causative agent, WM performance is improved by the exogenous administration of estradiol in treated animals [26•]. Although their presence was uncertain when our lab's work began, the presence of ER $\alpha$ receptors in DLPFC (a brain region heavily implicated in WM) has been confirmed in primate brains during the past decade, e.g., [42, 43]. For example, an immunolabeling study by Montague et al. [42] revealed abundant ER $\alpha$-positive cells throughout all layers of the DLPFC in the rhesus and human CNS. In female monkeys, dendritic spine densities within DLPFC have been discovered to vary as a function of both aging and circulating estradiol levels, and the density of a particular subtype of spine, the "thin" spine, is associated with cognitive performance in aged ovariectomized monkeys and is upregulated by a regimen of cyclic unopposed estradiol injections (for a review see [41•]). Estrogen-mediated changes in spine density are one candidate mechanism that has been proposed to explain estradiol's facilitative effects on WM [41•]. Recently, it was also discovered that the frequency of multisynaptic boutons in the DLPFC is positively correlated with accuracy on delayed response in NHP, and multisynaptic boutons are increased in aged ovariectomized female monkeys by cyclic treatment with estradiol [44] (although this model may more closely model surgical- than natural agerelated menopause).
Although effects on spine densities in PFC are likely to play an important role in WM function during aging, other candidate mechanisms also exist. These include the effects of circulating estradiol levels on frontal bioamines, including serotonergic [35], cholinergic [37], dopaminergic, or noradrenergic projections $[10,45]$. Rodent work demonstrates that activity in all of these pathways can be influenced by estradiol manipulations $[9,46]$, and each plays an important role in WM processes in primates [47]. Consequently, modulation of these pathways by available estradiol concentrations might underlie the WM effects observed, though no single transmitter is likely to be uniquely responsible on its own. For example, using a tryptophan depletion paradigm to lower central serotonin, Epperson et al. [35] found that tryptophan depletion attenuated activation in the DLPFC and cingulate gyrus during the two-back (high load) condition of an $N$-back task, which could be counteracted by estradiol treatment, suggesting estradiol's effects on WM are at least partially mediated by an action in serotonergic pathways. In other studies, postmenopausal women treated with estradiol showed increased frontal activation during high memory load conditions of an $\mathrm{N}$ back task compared to women who received placebo [36] and estradiol was found to counteract the effects of an anticholinergic drug (scopolamine) on BOLD activation in the medial frontal gyrus and inferior parietal lobule during $N$-back performance [37]. These findings suggest that estradiol may also act on the cholinergic system. Together, these findings taken in conjunction with the elegant work on spine densities in NHP imply that estradiol may facilitate frontocortical elements of the WM system by more than one route.

Frontal dopamine levels are important for WM function [47], especially spatial WM. The SPWM (Fig. 1) likely has significant dopamine variance as suggested by preliminary genotyping data from a small pilot study in which we found that the numbers of WM errors committed by women performing the SPWM could be statistically predicted based on their allelic variations in dopamine-related genes (COMT, $D R D 2$, but also NET) in conjunction with a common functional variant in the ER $\alpha$ gene, ESR1, based on two-loci association tests $[29,48]$. The number of WM errors produced on the SPWM has been found to vary as a function of the use of estrogen therapy in postmenopausal women [10], over the natural menstrual cycle in young women [23], and with estradiol concentrations during late pregnancy [29]. In a provocative fMRI study, Jacobs and d'Esposito [45] reported that individual variation in the catechol- $O$-methyltransferase gene (COMT) predicted women's accuracy on the $N$-back when considered in combination with estradiol levels over the menstrual cycle, suggesting a possible estradiol $\times$ genotype interaction (see also [49]). Although sample size for the critical comparisons was small, such an interaction in the effect of estradiol on WM might be expected if dopamine plays a significant contributing role, based on the known effects of the 
Val and Met alleles on basal dopamine concentration in the PFC [45]. Higher dopamine, inferred from high estradiol, Met genotype, or both, was associated with lower BOLD activation in the DLPFC bilaterally, interpreted by the authors as a reflection of more efficient neuronal recruitment. However, a definitive study showing an estradiol-dopamine link in humans is still lacking.

\section{Other Prefrontal Functions}

The evidence described above suggests that estradiol may promote the functioning of the WM system in aging women by modulating frontal regions involved in cognitive control. If estradiol is in fact active in PFC, its modulatory influence may extend beyond WM, as the dorsolateral cortex participates in a number of other executive functions including, for instance, attentional set-shifting (the ability to flexibly shift and then maintain one's attentional focus as new or previously unattended stimuli become behaviorally relevant, especially in the presence of distraction [47]). Few studies so far have addressed the possible endocrine regulation of other prefrontal processes, especially where aging populations are concerned. Recently, however, recruitment of the DLPFC was found to be enhanced during attentional set-shifting in early postmenopausal women who were treated with estradiol compared with placebo in an fMRI study that used a double-blind crossover design [50•]. This result is compatible with a female advantage recently reported on a measure of attentional shifting in younger adults < age 50 years [18]. Ovarian cycle-dependent effects on response inhibition, another function that evokes strong prefrontal recruitment [51,52], also have been reported $[53,54]$. While few in number, such findings imply that a modulatory effect of estradiol might be present not only for WM but also for other processes that depend heavily on the frontal cortex. Correspondingly, these processes too might be mildly and adversely affected by menopause in aging women (and potentially improved by estrogen therapies), although new research will be needed in order to test this hypothesis.

A recent study of prospective memory in postpartum women observed alterations in functional connectivity between frontoparietal sites and the hippocampus, evident using fMRI, which mediated an effect of circulating estradiol levels on prospective memory [55•]. Estradiol concentrations are extremely low and non-cyclic during the weeks following childbirth and resemble the concentrations seen after menopause. Collectively, such findings underscore the possibility that neuromodulatory effects of estradiol in the frontal cortex could be important for the maintenance of optimal neural functioning in women not only during aging but also during other major reproductive life events. Going forward, it will be important to assess more fully the range and limits of estrogen's effects in the female prefrontal cortex, and to understand the implications of estrogen loss under conditions where the production of estrogen is significantly decreased.

\section{Conclusions}

Many of the cognitive deficits associated with normal aging in humans (forgetfulness, reduced cognitive flexibility, distractibility) reflect diminished prefrontal function of the aging brain [56]. Some research suggests that age-associated decline in WM is an important mediator of age-related cognitive decline more generally, and in fact, controlling for WM function statistically attenuates the age-related declines observed in several other cognitive domains. NHPs are a useful animal model of WM given their close homologies with human brains [57]. With aging, NHP show a marked reduction of persistent neuronal firing in excitatory networks within the PFC that form a basis for WM, which can be rescued by restoring the appropriate neurochemical milieu [56]. In NHP, a disinhibition of cAMP signaling with aging may weaken thin spines in particular $[56,58]$, but changes occur in several relevant neurochemical pathways (e.g., [59]). A loss of the maintenance effects of estrogens in the PFC may be one important factor that contributes to diminished cognitive function and may disproportionately affect women, not only because of their higher estradiol levels during the reproductive years of the adult lifespan but also because, after menopause, men possess higher serum estradiol concentrations than women do (e.g., [60]). Whether or not estradiol plays a similar maintenance role in the male PFC is unknown. Men of course have higher testosterone (which serves as a substrate for estradiol production via aromatization), but the effect of testosterone on WM tasks requiring the participation of the PFC has received little study in men and preliminary findings are mixed (e.g., [61] but see $[62,63])$. Testosterone, too, decreases gradually but steadily with advancing age, and beyond age 70 years as many as $65 \%$ of men are hypogonadal [64].

The marked decline in ovarian steroid production after menopause may have consequences for women's cognitive function that extend beyond memory. However, memory (including WM) has been the most intensively studied to date. Research investigating the maintenance by estrogens of other prefrontal functions is still limited and future work ought to address a wider range of processes [65]. Because it is a key function intrinsic to the implementation of many other complex cognitive operations (e.g., behavioral planning, reading comprehension), a facilitative effect of estradiol on the efficiency of the prefrontal WM system (and its loss with aging) is an important finding that has broad repercussions for cognition. Any loss of function associated with diminished estrogens may have practical implications for optimizing the brain health of aging women. In addition, at a theoretical level, our emerging understanding of endocrine dysregulation within the 
CNS related to aging may generate new conceptual insights into basic processes that underlie the age-related declines seen in other functional domains.

It should be emphasized that the present review concerns normal aging, not the pathological processes associated with Alzheimer's disease (AD). However, lower endogenous estradiol levels compared with age-matched controls have been reported in women with actual or incipient Alzheimer's disease (e.g., [66]). It is unclear at the present time whether or not lower peripheral levels are reflected in the central CNS concentrations of women who have Alzheimer's [67] or whether replacement estrogens taken in the early postmenopause may help to protect against the accrual or progression of $\mathrm{AD}$ pathology $[68 \cdot, 69,70]$ in at-risk women.

In laboratory animals, estradiol promotes neurogenesis in the adult hippocampus, stimulates brain-derived neurotrophic factor and other growth factors, exerts regulatory effects in a number of neurochemical pathways, regulates microRNAs (and other epigenetic processes), and exerts protective effects on oxidative metabolism $[40,69,71]$. Although considered a part of ordinary aging, at least part of what we perceive to be age-related decline in frontocortical processes might actually be due to reduced circulating sex steroid concentrations and therefore at least partially remediable using well-tuned (and, potentially, well-timed) pharmacological interventions [72, 73]. In principle, the timing of interventions might matter in terms of their effectiveness although, to date, there is little support for a critical window concept as far as prefrontal function is concerned (e.g., [27, 74]), unlike the limited window of opportunity suggested to exist for the hippocampal effects of estrogens $[72,73]$. This difference might simply reflect a paucity of relevant data for WM, although a more interesting possibility is a true regional specificity in the temporal window of estradiol's effects.

Current guidelines suggest that hormone therapy started soon after menopause onset can offer specific health benefits for symptomatic women [75], but hormone therapy is not recommended at present as a means to treat or prevent cognitive decline in women who undergo natural age-related menopause [75]. This guideline is based largely on the equivocal evidence of cognitive benefit observed in studies that have investigated forms of memory dependent on the medial temporal lobe, not studies of WM and its neural substrates. Currently, the corpus of evidence supporting effects of estrogens on WM is still small, but accumulating data indicate that further study is warranted. In women who choose estrogens after menopause, treatment must be individualized to identify the most suitable type, dose, and formulation and weighed against a possible increase in health risks associated with longer-term use [75]. New intervention tools for menopausal women beyond conventional conjugated estrogens (e.g., Premarin) are under development. While low-dose estradiol continues to be tested [e.g., 70], promising alternative avenues for protecting women's brain health include simulating estradiol's effects on downstream molecular targets [71], newgeneration selective estrogen receptor modulators [76], or emerging estrogen prodrugs [77•] that have a potential to act selectively in the CNS without exerting undesirable proliferative effects in the breast or endometrium.

Acknowledgments Genotyping data reported in this article are courtesy of Dr. Wolfgang Sadée and Dr. Julia K. Pinsonneault (Ohio State University) who performed the genotyping analyses. I am grateful to Belal Howidi and Paul Istasy for referencing assistance. P. Istasy was the recipient of an NSERC Undergraduate Student Research Award.

Funding Information I thank the Natural Sciences and Engineering Research Council of Canada (NSERC) and the Society for Women's Health Research for partial funding of research reported here.

\section{Compliance with Ethical Standards}

Conflict of Interest The author declares the she has no conflict of interest.

Human and Animal Rights and Informed Consent This article does not contain any studies with human or animal subjects performed by any of the authors.

Open Access This article is distributed under the terms of the Creative Commons Attribution 4.0 International License (http:// creativecommons.org/licenses/by/4.0/), which permits unrestricted use, distribution, and reproduction in any medium, provided you give appropriate credit to the original author(s) and the source, provide a link to the Creative Commons license, and indicate if changes were made.

\section{References}

Papers of particular interest, published recently, have been highlighted as:

- Of importance

1. Daselaar S, Cabeza R. Age-related decline in working memory and episodic memory. In: Ochsner KN, Kosslyn S, editors. The Oxford handbook of cognitive neuroscience, Oxford handbooks online: Oxford University Press; 2013. https://doi.org/10.1093/oxfordhb/ 9780199988693.013.0022.

2. Braver TS, West R. Working memory, executive control and aging. In: Craik FIM, Salthouse TA, editors. The handbook of aging and cognition. 3rd ed. New York: Psychology Press; 2008. p. 311-72.

3. Funahashi S. Working memory in the prefrontal cortex. Brain Sci. 2017;7:49. https://doi.org/10.3390/brainsci7050049. A basic survey and theoretical overview of working memory and the frontal cortex, emphasizing primate research. A helpful overview for readers new to the field.

4. Kirchner WK. Age differences in short-term retention of rapidly changing information. J Exp Psychol. 1958;55:352-8.

5. Wiegersma S, van der Scheer E, Human R. Subjective ordering, short-term memory, and the frontal lobes. Neuropsychologia. 1990;28:85-98. https://doi.org/10.1016/0028-3932(90)90089-7. 
6. Owen AM, McMillan KM, Laird AR, Bullmore E. $N$-Back working memory paradigm: a meta-analysis of normative functional neuroimaging studies. Hum Brain Mapp. 2005;25:46-59. https:// doi.org/10.1002/hbm.20131.

7. Barbey AK, Koenigs M, Grafman J. Dorsolateral prefrontal contributions to human working memory. Cortex. 2013;49:1195-205. https://doi.org/10.1016/j.cortex.2012.05.022.

8. Hackman BW, Galbraith D. Replacement therapy and piperazine oestrone sulphate ('Harmogen') and its effect on memory. Curr Med Res Opin. 1976;4:303-6.

9. Shanmugan S, Epperson CN. Estrogen and the prefrontal cortex: towards a new understanding of estrogen's effects on executive functions in the menopause transition. Hum Brain Mapp. 2014;35:847-65. https://doi.org/10.1002/hbm.22218.

10. Duff SJ, Hampson E. A beneficial effect of estrogen on working memory in postmenopausal women taking hormone replacement therapy. Horm Behav. 2000;38:262-76. https://doi.org/10.1006/ hbeh.2000.1625.

11. Keenan PA, Ezzat WH, Ginsburg K, Moore GJ. Prefrontal cortex as the site of estrogen's effect on cognition. Psychoneuroendocrinology. 2001;26:577-90

12. Hampson E. Estrogens and androgens in the prefrontal cortex: relevance for cognition and decision-making. In: Schultheiss OC, Mehta PH, editors. International handbook of social neuroendocrinology. Abingdon: Routledge Psychology Press. in press.

13. Roberts JA, Gilardi KVK, Lasley B, Rapp PR. Reproductive senescence predicts cognitive decline in aged female monkeys. NeuroReport. 1997;8:2047-51.

14. Duff SJ, Hampson E. A sex difference on a novel spatial working memory task in humans. Brain Cogn. 2001;47:470-93. https://doi. org/10.1006/brcg.2001.1326.

15. Lejbak L, Vrbancic M, Crossley M. The female advantage in object location memory is robust to verbalizability and mode of presentation of test stimuli. Brain Cogn. 2009;69:148-53. https://doi.org/10. 1016/j.bandc.2008.06.006.

16. Robert M, Savoie N. Are there gender differences in verbal and visuospatial working-memory resources? Eur J Cog Psychol. 2006;28:378-97. https://doi.org/10.1080/09541440500234104.

17. McDowell AA, Brown WL, McTee AC. Sex as a factor in spatial delayed-response performance by rhesus monkeys. J Comp Physiol Psychol. 1960;53:420-32. https://doi.org/10.1037/h0043372.

18. Kuptsova SV, Ivanova MV, Petrushevsky AG, Fedina ON, Zhavoronkova LA. Sex-related differences in task-switching: an fMRI study. Hum Physiol. 2015;41:611-24. https://doi.org/10. 1134/S0362119715050084

19. Chechlacz M, Rotshtein P, Humphreys GW. Neuronal substrates of Corsi Block span: Lesion symptom mapping analyses in relation to attentional competition and spatial bias. Neuropsychologia 2014: 64:240-251. Doi: https://doi.org/10.1016/j.neuropsychologia. 2014.09.0380028-3932.

20. Reed JL, Gallagher NM, Sullivan M, Callicott JH, Green AE. Sex differences in verbal working memory performance emerge at very high loads of common neuroimaging tasks. Brain Cogn. 2017;113: 56-64. https://doi.org/10.1016/j.bandc.2017.01.001. Among groups of participants matched on their level of performance at low WM loads, sex differences still emerged at high WM loads, with men showing higher accuracy than women. Two versions of the $\mathrm{N}$-back task were used.

21. Voyer D, Voyer SD, Saint-Aubin J. Sex differences in visual-spatial working memory: a meta-analysis. Psychon Bull Rev. 2017;24: 307-34. https://doi.org/10.3758/s13423-016-1085-7. A metaanalysis looking at the question of whether there are sex differences in accuracy on several categories of non-verbal tasks that purport to assess $\mathrm{WM}$.

22. Jaeggi SM, Buschkuehl M, Perrig WJ, Meier B. The concurrent validity of the N-back task as a working memory measure.
Memory. 2010;18:394-412. https://doi.org/10.1080/ 09658211003702171.

23. Hampson E, Morley EE. Estradiol concentrations and working memory performance in women of reproductive age. Psychoneuroendocrinology. 2013;38:2897-904. https://doi.org/10. 1016/j.psyneuen.2013.07.020.

24. Tinkler GP, Voytko ML. Estrogen modulates cognitive and cholinergic processes in surgically menopausal monkeys. Prog NeuroPsychopharmacol Biol Psychiatry. 2005;29:423-31. https://doi. org/10.1016/j.pnpbp.2004.12.016.

25. Rapp PR, Morrison JH, Roberts JA. Cyclic estrogen replacement improves cognitive function in aged ovariectomized rhesus monkeys. J Neurosci. 2003;23:5708-14. https://doi.org/10.1523/ JNEUROSCI.23-13-05708.2003.

26. Kohama SG, Renner L, Landauer N, Weiss AR, Urbanski HF, Park $\mathrm{B}$, et al. Effect of ovarian hormone therapy on cognition in the aged female rhesus macaque. J Neurosci. 2016;36:10416-24. https://doi. org/10.1523/JNEUROSCI.0909-16.2016. During repeated testing over a 12-month period, ovariectomized female macaques with estradiol implants performed consistently better on a delayed response test of WM than all other groups. These data demonstrate that chronic sustained estradiol has beneficial effects on the functioning of the WM system.

27. Krug R, Born J, Rasch B. A 3-day estrogen treatment improves prefrontal cortex-dependent cognitive function in postmenopausal women. Psychoneuroendocrinology. 2006;31:965-75. https://doi. org/10.1016/j.psyneuen.2006.05.007.

28. Passingham RE. Memory of monkeys (Macaca mulatta) with lesions in prefrontal cortex. Behav Neurosci. 1985;99:3-21. https:// doi.org/10.1037/0735-7044.99.1.3.

29. Hampson E, Phillips SD, Duff-Canning SJ, Evans KL, Merrill M, Pinsonneault JK, et al. Working memory in pregnant women: relation to estrogen and antepartum depression. Horm Behav. 2015;74: 218-27. https://doi.org/10.1016/j.yhbeh.2015.07.006.

30. Romine CB, Reynolds CR. Sequential memory: a developmental perspective on its relation to frontal lobe functioning. Neuropsychol Rev. 2004;14:43-64.

31. Chen X, He X, Tao L, Li J, Wu J, Zhu C, et al. The working memory and dorsolateral prefrontal-hippocampal functional connectivity changes in long-term survival breast cancer patients treated with tamoxifen. Int J Neuropsychopharmacol. 2017;20:374-82. https://doi.org/10.1093/ijnp/pyx008.

32. Grigorova M, Sherwin BB, Tulandi T. Effects of treatment with leuprolide acetate depot on working memory and executive functions in young premenopausal women. Psychoneuroendocrinology. 2006;31:935-47. https://doi.org/10.1016/j.psyneuen.2006.05.004.

33. Islam F, Sparkes C, Roodenrys S, Astheimer L. Short-term changes in endogenous estrogen levels and consumption of soy isoflavones affect working and verbal memory in young adult females. Nutr Neurosci. 2008;11:251-62. https://doi.org/10.1179/ $147683008 X 301612$.

34. Leeners B, Kruger THC, Geraedts K, Tronci E, Mancini T, Ille F, et al. Lack of associations between female hormone levels and visuospatial working memory, divided attention and cognitive bias across two consecutive menstrual cycles. Front Behav Neurosci. 2017;11: 120. https://doi.org/10.3389/fnbeh.2017.000120.

35. Epperson CN, Amin Z, Ruparel K, Gur R, Loughead J. Interactive effects of estrogen and serotonin on brain activation during working memory and affective processing in menopausal women. Psychoneuroendocrinology. 2012;37:372-82. https://doi.org/10. 1016/j.psyneuen.2011.07.007.

36. Dumas JA, Kutz AM, Naylor MR, Johnson JV, Newhouse PA. Increased memory load-related frontal activation after estradiol treatment in postmenopausal women. Horm Behav. 2010;58:929 35. https://doi.org/10.1016/j.yhbeh.2010.09.003. 
37. Dumas JA, Kutz AM, Naylor MR, Johnson JV, Newhouse PA. Estradiol treatment altered anticholinergic-related brain activation during working memory in postmenopausal women. Neuroimage. 2012;60:1394-403. https://doi.org/10.1016/j.neuroimage.2012.01. 043.

38. Smith YR, Love T, Persad CC, Tkaczyk A, Nichols TE, Zubieta JK. Impact of combined estradiol and norethindrone therapy on visuospatial working memory assessed by functional magnetic resonance imaging. J Clin Endocrinol Metab. 2006;91:4476-81. https://doi. org/10.1210/jc.2006-0907.

39. Jacobs EG, Weiss B, Makris N, Whitfield-Gabrieli S, Buka SL, Klibanski A. Reorganization of functional networks in verbal working memory circuitry in early midlife: the impact of sex and menopausal status. Cereb Cortex. 2017;27:2857-70. https://doi.org/10. 1093/cercor/bhw127. Re-organization of the functional networks underlying WM was evident as differences in taskrelated activation in DLPFC and hippocampus in postmenopausal women compared with premenopausal women.

40. Galea LAM, Frick KM, Hampson E, Sohrabji F, Choleris E. Why estrogens matter for behavior and brain health. Neurosci Biobehav Rev. 2017;76:363-79.

41. Hara Y, Waters EM, McEwen BS, Morrison JH. Estrogen effects on cognitive and synaptic health over the lifecourse. Physiol Rev. 2015;95:785-807. https://doi.org/10.1152/physrev.00036.2014. This article summarizes a set of studies by the authors showing effects of estrogens on spinogenesis and synaptogenesis in the DLPFC (BA 46) of young and aged female monkeys.

42. Montague D, Weickert CS, Tomaskovic-Crook E, Rothmond DA, Kleinman E, Rubinow DR. Oestrogen receptor $\alpha$ localisation in the prefrontal cortex of three mammalian species. J Neuroendocrinol. 2008;20:893-903.

43. Wang AC, Hara Y, Janssen WG, Rapp PR, Morrison JH. Synaptic estrogen receptor- $\alpha$ levels in prefrontal cortex in female rhesus monkeys and their correlation with cognitive performance. $\mathrm{J}$ Neurosci. 2010;30:12770-6. https://doi.org/10.1523/jneurosci. 3192-10.2010.

44. Hara Y, Yuk F, Puri R, Janssen WG, Rapp PR, Morrison JH. Estrogen restores multisynaptic boutons in the dorsolateral prefrontal cortex while promoting working memory in aged rhesus monkeys. J Neurosci. 2016;36:901-10. https://doi.org/10.1523/ jneurosci.3480-13.2016

45. Jacobs E, D'Esposito M. Estrogen shapes dopamine-dependent cognitive processes: implications for women's health. J Neurosci. 2011;31:5286-93. https://doi.org/10.1523/JNEUROSCI.6394-10. 2011.

46. Barth C, Villringer A, Sacher J. Sex hormones affect neurotransmitters and shape the adult female brain during hormonal transition periods. Front Neurosci. 2015;9:37. https://doi.org/10.3389/fnins. 2015.00037

47. Robbins TW, Arnsten AFT. The neuropsychopharmacology of fronto-executive function: monoaminergic modulation. Annu Rev Neurosci. 2009;32:267-87. https://doi.org/10.1146/annurev.neuro. 051508.135535 .

48. Hampson E, Pinsonneault J, Steiner M, Sadee W. The neurochemistry of spatial working memory: insights from genetic association analysis. Front Hum Neurosci. 2010;4. https://doi.org/10.3389/ conf.fnins.2010.14.00053.

49. Papaleo F, Sannino S, Piras F, Spalletta G. Sex-dichotomous effects of functional COMT genetic variations on cognitive functions disappear after menopause in both health and schizophrenia. Eur Neuropsychopharmacol. 2015;25:2349-63. https://doi.org/10. 1016/j.euroneuro.2015.10.005.

50. Girard R, Météreau E, Thomas J, Pugeat M, Qu C, Dreher JC. Hormone therapy at early post-menopause increases cognitive control-related prefrontal activity. Sci Rep 2017:7:44917. https:// doi.org/10.1038/srep44917. Estradiol treatment enhanced DLPFC recruitment during an attentional switching task. A double-blind, placebo-controlled, crossover design was used.

51. Oldrati V, Patricelli J, Colombo B, Antonietti A. The role of dorsolateral prefrontal cortex in inhibition mechanism: a study on cognitive reflection test and similar tasks through neuromodulation. Neuropsychologia. 2016;91:499-508. https://doi.org/10.1016/j. neuropsychologia.2016.09.010.

52. He Q, Chen M, Chen C, Xue G, Feng T, Bechara A. Anodal stimulation of the left DLPFC increases IGT scores and decreases delay discounting rate in healthy males. Front Psychol. 2016;7:1421. https://doi.org/10.3389/fpsyg.2016.01421.

53. Smith CT, Sierra Y, Oppler SH, Boettiger CA. Ovarian cycle effects on immediate reward selection bias in humans: a role for estradiol. J Neurosci. 2014;34:5468-76. https://doi.org/10.1523/jneurosci. 0014-14.2014.

54. Diekhof EK. Be quick about it. Endogenous estradiol level, menstrual cycle phase and trait impulsiveness predict impulsive choice in the context of reward acquisition. Horm Behav. 2015;74:186-93. https://doi.org/10.1016/j.yhbeh.2015.06.001.

55. Shin NY, Bak Y, Nah Y, Han S, Kim DJ, Kim SJ, et al. Disturbed retrieval network and prospective memory decline in postpartum women. Sci Rep. 2018;8:5476. https://doi.org/10.1038/s41598018-23875-5. Prospective memory was poorer in the postpartum period and estradiol levels were found in a mediation analysis to have an indirect effect on task accuracy via decreased functional connectivity between the hippocampus and ventral frontoparietal network.

56. Wang M, Gamo NJ, Yang Y, Jin LE, Wang XJ, Laubach M, et al. Neuronal basis of age-related working memory decline. Nature. 2011;476:210-3. https://doi.org/10.1038/nature10243.

57. Passingham R. How good is the macaque monkey model of the human brain? Curr Opin Neurobiol. 2009;19:6-11. https://doi.org/ 10.1016/j.conb.2009.01.002.

58. Arnsten AFT, Paspalas CD, Gamo NJ, Yang Y, Wang M. Dynamic network connectivity: a new form of neuroplasticity. Trends Cogn Sci. 2010;14:365-75. https://doi.org/10.1016/j.tics.2010.05.003.

59. Newhouse P, Dumas J. Estrogen-cholinergic interactions: implications for cognitive aging. Horm Behav. 2015;74:173-85. https:// doi.org/10.1016/j.yhbeh.2015.06.022.

60. Carlson LE, Sherwin BB, Chertkow HM. Relationship between mood and estradiol $\left(\mathrm{E}_{2}\right)$ levels in Alzheimer's disease (AD) patients. J Gerontol B Psychol Sci Soc Sci. 2000;55:P47-53.

61. Janowsky JS, Chavez B, Orwoll E. Sex steroids modify working memory. J Cogn Neurosci. 2000;12:407-14. https://doi.org/10. 1162/089892900562228.

62. Alibhai SMH, Mahmoud S, Hussain F, Naglie G, Tannock I, Tomlinson G, et al. Levels of sex hormones have limited effect on cognition in older men with or without prostate cancer. Crit Rev Oncol Hematol. 2010;73:167-75.

63. Hajszan T, MacLusky NJ, Leranth C. Role of androgens and the androgen receptor in remodeling of spine synapses in limbic brain areas. Horm Behav. 2008;53:638-46. https://doi.org/10.1016/j. hybeh.2007.12.007.

64. Mitchell S, Harman E, Metter J, Tobin JD, Pearson J, Blackman MR. Longitudinal effects of aging on serum total and free testosterone levels in healthy men. J Clin Endocrinol Metab. 2001;86: 724-31. https://doi.org/10.1210/jcem.86.2.7219.

65. Evans KL, Hampson E. Sex differences on prefrontally-dependent cognitive tasks. Brain Cogn. 2015;93:42-53. https://doi.org/10. 1016/j.bandc.2014.11.006.

66. Manly JJ, Merchant CA, Jacobs DM, Small SA, Bell K, Ferin M, et al. Endogenous estrogen levels and Alzheimer's disease among postmenopausal women. Neurology. 2000;54:833-7.

67. Honma N, Saji S, Mikami T, Yoshimura N, Mori S, Saito Y, et al. Estrogen-related factors in the frontal lobe of Alzheimer's disease 
patients and importance of body mass index. Sci Rep. 2017;7:726. https://doi.org/10.1038/s41598-017-00815-3.

68. Imtiaz B, Tuppurainen M, Rikkonen T, Kivipelto M, Soininen H, Kröger H, et al. Postmenopausal hormone therapy and Alzheimer's disease: a prospective cohort study. Neurology. 2017;88:1062-8. https://doi.org/10.1212/WNL.0000000000003696. Twenty-year follow-up data from a large Finnish sample showed that postmenopausal estrogen use was not associated with Alzheimer's disease risk, although self-reported long-term use ( $>10$ years) was associated with a reduction in $\mathrm{AD}$ risk compared with nonusers.

69. Merlo S, Spampinato SF, Sortino MA. Estrogen and Alzheimer's disease: still an attractive topic despite disappointment from early clinical results. Eur J Pharmacol. 2017;817:51-8. https://doi.org/10. 1016/j.ejphar.2017.05.059.

70. Kantarci K, Lowe VJ, Lesnick TG, Tosakulwong N, Bailey KR, Fields JA, et al. Early postmenopausal transdermal 17beta-estradiol therapy and amyloid-beta deposition. J Alzheimers Dis. 2016;53: 547-56.

71. Frick KM. Molecular mechanisms underlying the memoryenhancing effects of estradiol. Horm Behav. 2015;74:4-18. https://doi.org/10.1016/j.yhbeh.2015.05.001.

72. Smith CC, Vedder LC, Nelson AR, Bredemann TM, McMahon LL. Duration of estrogen deprivation, not chronological age, prevents estrogen's ability to enhance hippocampal synaptic physiology.
Proc Natl Acad Sci U S A. 2010;107:19543-8. https://doi.org/10. 1073/pnas.1009307107.

73. Maki PM. Critical window hypothesis of hormone therapy and cognition: a scientific update on clinical studies. Menopause. 2013;20:695-709.

74. Baxter MG, Santistevan AC, Bliss-Moreau E, Morrison JH. Timing of cyclic estradiol treatment differentially affects cognition in aged female rhesus monkeys. Behav Neurosci. 2018;132:213-23. https://doi.org/10.1037/bne0000259.

75. North American Menopause Society. The 2017 hormone therapy position statement of the North American Menopause Society. Menopause. 2017;24:728-53.

76. Luine VN. Estradiol and cognitive function: past, present and future. Horm Behav. 2014;66:602-18. https://doi.org/10.1016/j. yhbeh.2014.08.011

77. Prokai L, Nguyen V, Szarka S, Garg P, Sabnis G, Bimonte-Nelson HA, et al. The prodrug DHED selectively delivers $17 \beta$-estradiol to the brain for treating estrogen-responsive disorders. Sci Transl Med. 2015;7:297ra113. https://doi.org/10.1126/scitranslmed.aab1290. Demonstrated that a small molecule precursor prodrug converted to $17 \beta$-estradiol in the brains of rats after systemic administration while remaining inert in the rest of the body and elicited changes in gene expression and neuronal morphology that mirrored those typically seen after direct $17 \beta$-estradiol treatment. 